Journal of Engineering and Applied Sciences 15 (6): 1468-1472, 2020

ISSN: 1816-949X

(C) Medwell Journals, 2020

\title{
Rorschach Inkblot Image Stylization using Silhouette Matching and Paper Texture Simulation
}

\author{
Jong Chul Yoon \\ Department of Software and Media Engineering, Kangwon National University, \\ Chuncheon, South Korea
}

\begin{abstract}
The Rorschach inkblot image is a monochrome or color image of the ink stain used in psychological testing. It is used to predict human psychology projected on a vague image that can be interpreted in various shapes. In this study, we introduce a system that automatically generates a Rorschach image by automatically selecting a similar form in a silhouette image database. Using the results of this study, it is possible to generate various types of Rorschach images and it can be used novel Non-Photorealistic Rendering (NPR) application.
\end{abstract}

Key words: Rorschach, color image, psychological, automatically, NPR, introduce

\section{INTRODUCTION}

The Rorschach test (Exner and Erdberg, 2002) was developed by Rorschach in 1921 and it is used to interpret human tendencies or analyze emotions by using the ten ink stains shapes represented in Fig. 1. Various scholars have provided a methodology for analyzing and extending Rorschach tests and these studies are widely used as a method for psychological testing in current psychoanalysis. However, for using these inkblot cards, the user must purchase predefined images and there is a limitation that can not be easily produced without an expert.

In this study, we introduce an automatic Rorschach image styling technique that uses a shape matching between user-specified silhouette images. The Rorschach image can be defined as an image that contains various types of silhouettes coexist in a single image and that can be regarded as a type of ambiguous art (Mitra et al., 2009). We aim to create an ambiguous optical result that the computer automatically generates which has the benefit of drastically reducing the cost of producing output. To develop an automated system, we first construct a database of simplified silhouette images input from users and analyze them to extract shape generators for each silhouette. The extracted shape constructor is used to match the silhouette database and the silhouette of the input image. Auto-correlation-based matching is additionally performed for accurate matching. The pixel-based abstraction of overlapping images is then performed to generate the final Rorschach image. An overview of our system is shown in Fig. 2.

Literature review: Existing methods for image stylization mainly focus on the aesthetic improvement of an image. For example, stroke-based rendering techniques (Hertzmann, 2001; Gooch et al., 2002, Durand et al., 2001, Hays and Essa, 2004), convert image to a non-photorealistic style by filling its region with certain types of strokes. Image abstraction or cartooning (DeCarlo and Santella, 2002; Wang et al., 2004; Winnemoller et al., 2006) provides stylization via image segmentation and line drawing. Rorschach image styling method proposed in this study can be considered as an (a)

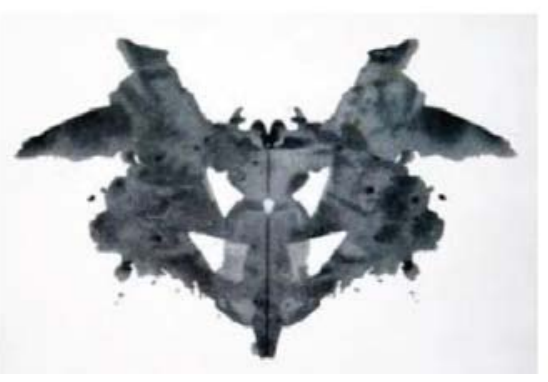

(b)

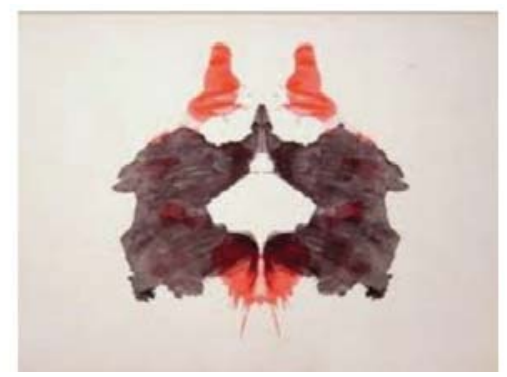

Fig. 1(a, b): Rorschach ink blot card (Exner and Erdberg, 2002) 


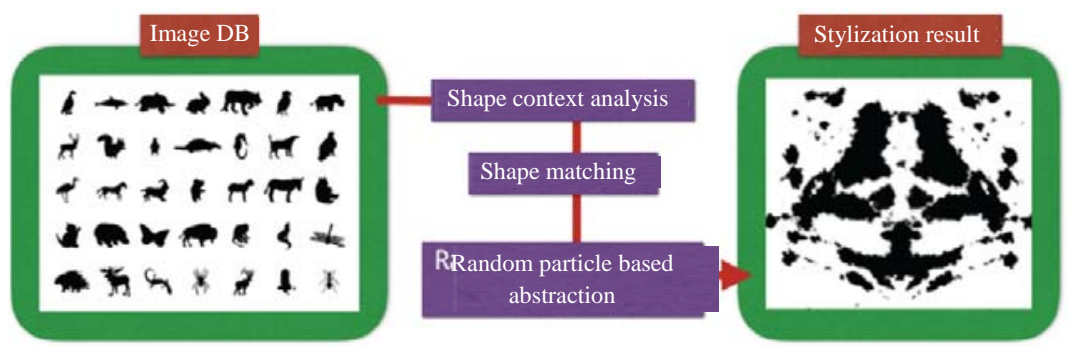

Fig. 2: System overview

image conversion technique that has a specific purpose, not an artistic effect, among existing non-realistic rendering techniques. It has the significance of overcoming the shortcomings of impracticality which is a limitation of existing non-realistic rendering techniques.

\section{MATERIALS AND METHODS}

Silhouette image analysis: To construct the silhouette image database, the image data based is constructed by collecting vector images distributed freely on the internet. Since, we do not use the color information of the image but only use shapes, we convert all of the images into binarized images (Fig. 2). General Rorschach images constructed by a combination of shape by animal or human, database consists of human poses and animal forms. We constructed a database using a total of 100 images. The purpose of the proposed system is to overlap two or more similar forms. Therefore, it is necessary to define shape descriptors that can determine shape similarity. The shape descriptors used in our system are based on the concept of shape context (Yoon et al., 2011) which abstracts the shape of a point cloud. The shape context consists of a small number of histograms that express the spatial relationships among the points representing the shape and allow a possible match between two given shapes to be evaluated quickly. The use of directional relationships and relative distances between the points makes the shape context scale and translation-invariant. Furthermore, Principal Components Analysis (PCA) based axis adaptation can help obtain a rotation-invariant shape context. We first extract the feature points from the silhouette image using a well-known Harris corner detection method (Wahba, 1990) (Fig. 3a), then, construct 32 bins (Fig. 3b) and calculate the histograms of each image in silhouette database.

The analyzed shape context has the form of a one-dimensional vector and can be used to analyze whether the silhouette has a symmetrical shape or not. In the case of Rorschach images, they are generally, horizontally symmetrical. Therefore, in the case of symmetrical shapes input, one side is removed and forcedly convert to symmetrical shape.
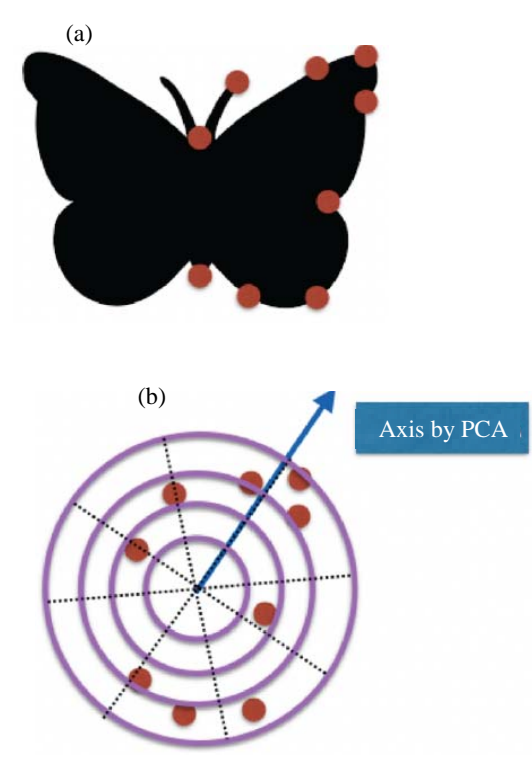

Fig. 3(a, b): Feature points extraction and shape constructor for the input silhouette image (a) For symmetrical silhouette images, we use only one side to extract feature points and (b) Rotation-invariant shape context with 32 bins which represents a shape as the number of feature points in the bin

Figure 4 shows similar pairs extracted by the dot product. Although, the shape context we used is invariant to rotation, for more accurate orientation matching, we compute the auto-correlation between shape context which has a vector form and fid the better location between the shapes by minimized the auto-correlation value (Fig. 4c).

Computing similarity: We now compare all possible pairs of shape contexts in the database. To find the best match, we calculate the similarity between each pair of shape contexts independently. A shape context is represented by an array of values, each of which corresponds to the number of points in a histogram bin. Therefore, we can calculate the similarity between two shape contexts by using a computation similar to a dot product. 
(a)

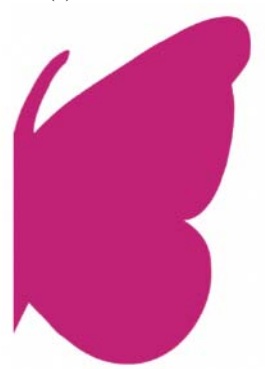

(b)

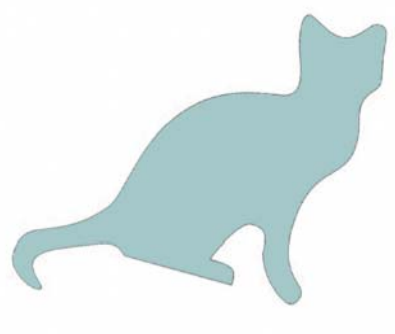

(c)

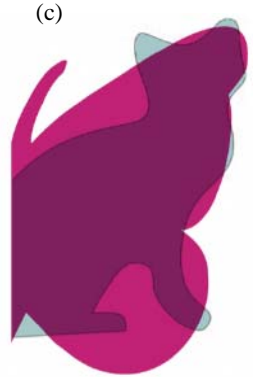

Fig. 4(a-c): (a) Secondary silhouette matching (b) Selected pair that has a similar shape using the shape context and (c) For detailed matching, local merge is performed to minimize the auto-correlation value

(a)

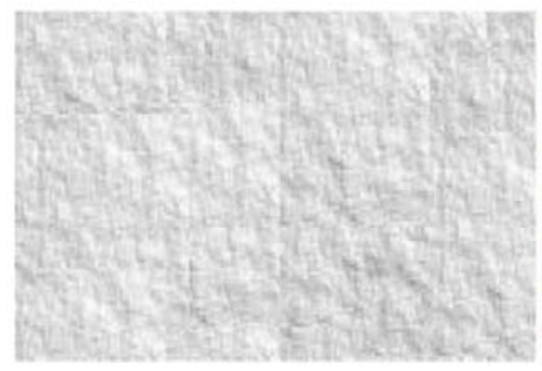

(b)

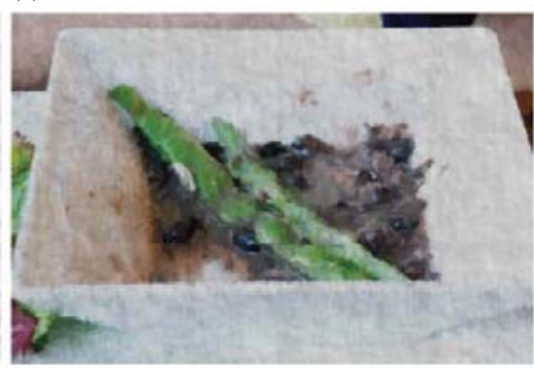

Fig. 5(a, b): Paper texture mapping combined with a quasi-painterly rendering, (a) The input paper texture and (b) The stylization result produced by the perturbed 2D flow field

(a)

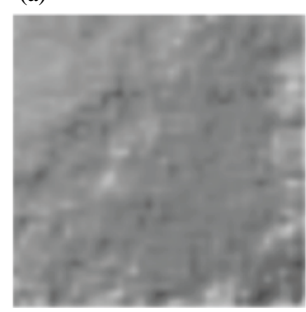

(b)

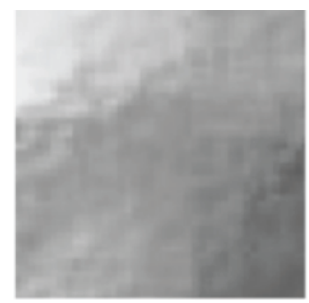

Fig. 6(a, b): Pattern regeneration using the biased noise function: to extract the random pattern: we use 5 octave noise functions with $128 \times 128$ sized gradient table using the hierarchical noise fiting method

Ink-like stylization: A general Rorschach image has an abstracted ink form. To simulate this, we stylized the overlapped image using pixel-simulation based abstraction technique that is based on Edge Tangent Flow (ETF). To generate an ink-like image, the paper texture should be considered for enhancing the drawing-like effect. To generate the paper texture effect, we design an image stylization system that perturbs the 2D flow field using the normal map which is commonly used to model a paper texture. We first extract the normal vectors of paper texture using a procedural noise function and then apply it for perturbing the 2D flow field which will be used as the directional field for particle simulation-based

stylization. Figure 5 shows a simple example of a painterly rendered image that combines with paper texture. To effectively express the ink bleeding effect, we applied random numbers to the ETFs of each shape image separately and then executed pixel simulations. We use real paper images as input for random numbers. Generally, paper texture consists of random patterns which have various frequencies. Therefore, a procedural noise function can be used to extract the random patterns and can also extend the pattern to a large domain that can cover sufficient domain for image resolution. We first extract the representative frequency of a real paper texture using the method. Since the real paper texture contains a list of a random signal which has different frequencies, we apply the hierarchical pattern extraction method. Regenerated textures by the extracted procedural noise function are shown in Fig. 6. Let $\mathrm{N}(\mathrm{x})$ be the biased noise function that is extracted from a real paper texture. Then, a perturbed flow vector field $\mathrm{F}^{\prime}(\mathrm{x})$ is calculated as follows:

$$
\mathrm{F}^{\prime}(\mathrm{x})=\mathrm{F}(\mathrm{x})+\mathrm{g} \mathrm{N}(\mathrm{x})
$$

where, $\mathrm{F}(\mathrm{x})$ is ETF of an input silhouette image and $\mathrm{g}$ is a significant weight. Using the perturbed flow vector field $\mathrm{F}^{\prime}(\mathrm{x})$, we can simulate the pixel particle to generate an ink-like image. The system is designed to generate various types of results by providing an interface that can give brightness and color difference between silhouettes according to user selection. 
(a)

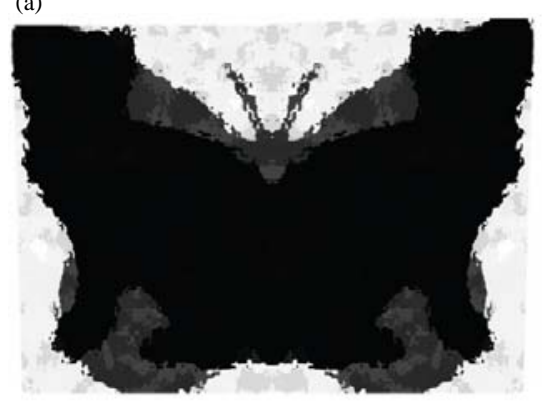

(b)

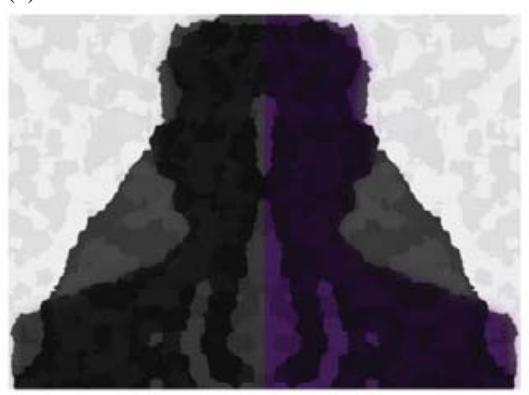

Fig. 7(a, b): Rorschach stylization results using two silhouettes, (a) Rorschach image using butterfly and cat; Brightness difference and (b) Rorschach image using people and dogs; Color different

\section{RESULTS AND DISCUSSION}

Figure 7 shows the results of Rorschach image stylization which created by two different silhouettes using our system. In Fig. 7a, result image can be interpreted by two shapes; cat and butterfly. To be able to distinguish between the phases, brightness or color differences were assigned to produce ambiguous but distinguishable results. The highest level of output has been achieved when a symmetrical image is chosen, due to the characteristics of the Rorschach image. To test our system, we composed ten college students into the experimental group who do not has experience with the Rorschach image to measure the accuracy of the hidden object. A total of five Rorschach images with two silhouettes hidden were made and provided to the tester to describe the shapes seen from the images. As a result of the evaluation, 66\% tester was accurately inferring silhouette images and a 25\% tester infer a completely different form. This mistake is considered meaningful in that it creates a new image according to the psychology of people which is the original purpose of the Rorschach image.

\section{CONCLUSION}

In this study, we presented that the new image styling technique, Rorschach image generation technique and this approach is considered to be beyond the limits of the impracticality of existing non-photo realistic rendering. The stable results have been achieved when we use two shapes of synthesis and when we use three or more silhouettes are included there was a limit of expression if there was no variation of contrast or color. The future goal is to automate these user choices and generate ambiguity illusion results that contain more diverse forms.

\section{ACKNOWLEDGEMENT}

This study is supported by 2016 Research Grant from Kangwon National University (No. 620160132) and Basic Science Research Program through the National Research Foundation of Korea (NRF) funded by the Ministry of Education (NRF-2019R1I1A3A01061157).

\section{REFERENCES}

DeCarlo, D. and A. Santella, 2002. Stylization and abstraction of photographs. ACM Trans. Graph., 21: 769-776.

Durand, F., V. Ostromoukhov, M. Miller, F. Duranleau and J. Dorsey, 2001. Decoupling strokes and high-level attributes for interactive traditional drawing. Proceedings of the Eurographics Workshop on Rendering Techniques, June 25-27, 2001, London, UK., pp: 71-82.

Exner Jr, J.E. and P. Erdberg, 2002. The Rorschach, Basic Foundations and Principles of Interpretation. 4th Edn.,/Vol. 1, Wiley, Hoboken, New Jersey, USA., ISBN: 9780471386728, Pages: 696.

Gooch, B., G. Coombe and P. Shirley, 2002. Artistic vision: Painterly rendering using computer vision techniques. Proceedings of the 2nd International Symposium on Non-Photorealistic Animation and Rendering (NPAR'02), June 03-05, 2002, ACM, Annecy, France, pp: 83-90.

Hays, J. and I. Essa, 2004. Image and video based painterly animation. Proceedings of the 3rd International Symposium on Non-Photorealistic Animation and Rendering (NPAR'04), June 07-09, 2004, ACM, Annecy, France, pp: 113-120.

Hertzmann, A., 2001. Paint by relaxation. Proceedings of the 2001 International Conference on Computer Graphics, July 6, 2001, IEEE, Hong Kong, China, pp: 47-54.

Mitra, N.J., H.K. Chu, T.Y. Lee, L. Wolf, H. Yeshurun and D. Cohen-Or, 2009. Emerging images. ACM. Trans. Graphics (TOG), Vol. 28, No. 5. 10.1145/1618452.1618509 
Wahba, G., 1990. Spline Models for Observational Data. 1st Edn., SIAM, Philadelphia, Pennsylvania, USA., ISBN: 10: 0898712440, Pages: 180.

Wang, J., Y. Xu, H.Y. Shum and M.F. Cohen, 2004. Video toning. Proceedings of the International Conference on ACM SIGGRAPH (SIGGRAPH'04), August 08-12, 2004, Los Angeles, California, USA., pp: 574-583.
Winnemoller, H., S.C. Olsen and B. Gooch, 2006. Real-time video abstraction. ACM Trans. Graph., 25: 1221-1226.

Yoon, J.C., I.K. Lee and H. Kang, 2011. Video painting based on a stabilized time-varying flow field. IEEE. Trans. Visual. Comput. Graphics, 18: 58-67. 